\title{
Frequência dos principais agentes infecciosos em pacientes queimados: uma revisão integrativa
}

\author{
Frequency of the main infectious agents in burn patients: an integrative review \\ Frecuencia de los principales agentes infecciosos en pacientes quemados: una revisión integradora
}

Recebido: 23/09/2021 | Revisado: 30/09/2021 | Aceito: 01/10/2021 | Publicado: 04/10/2021

\author{
Allef Francisco Lira da Rocha Braga \\ ORCID: https://orcid.org/0000-0001-7326-0012 \\ Universidade Tiradentes, Brasil \\ E-mail: allefbraga@gmail.com \\ Gabriel Ponciano Santos de Carvalho \\ ORCID: https://orcid.org/0000-0001-7476-7907 \\ Universidade Tiradentes, Brasil \\ E-mail: gabriel.ponciano19@gmail.com \\ Luiz Felipe Santos Dias \\ ORCID: https://orcid.org/0000-0001-7971-9381 \\ Universidade Tiradentes, Brasil \\ E-mail: luiz.dias@souunit.com.br \\ Aline Sales Pessoa Fleury \\ ORCID: https://orcid.org/0000-0003-4193-8049 \\ Universidade Tiradentes, Brasil \\ E-mail: alinespf1302@gmail.com \\ Wesley Melo de Santana \\ ORCID: https://orcid.org/0000-0003-3863-5821 \\ Universidade Tiradentes, Brasil \\ E-mail: wesley.melo@ souunit.com.br \\ Sidcley Felix dos Santos \\ ORCID: https://orcid.org/0000-0003-3819-2186 \\ Universidade Tiradentes, Brasil \\ E-mail: sidcley_felix@ hotmail.com \\ Nathalia Barretto dos Santos \\ ORCID: https://orcid.org/0000-0002-8148-5737 \\ Universidade Tiradentes, Brasil \\ E-mail: nathaliabarretto1@gmail.com \\ Patrícia Santos Silva \\ ORCID: https://orcid.org/0000-0001-5590-9658 \\ Universidade Tiradentes, Brasil \\ E-mail: 4patriciasantos@gmail.com \\ Wianne Santos Silva \\ ORCID: https://orcid.org/0000-0001-8006-8934 \\ Universidade Tiradentes, Brasil \\ E-mail: wiannessilva@gmail.com \\ Kellyn Mariane Souza Sales \\ ORCID: https://orcid.org/0000-0001-6233-5103 \\ Universidade Tiradentes, Brasil \\ E-mail: kellynsales1@gmail.com \\ Bruno Barreto Cintra \\ ORCID: https://orcid.org/0000-0003-3835-1968 \\ Universidade Tiradentes, Brasil \\ E-mail: bbcintra@doctor.com
}

\section{Resumo}

Objetivo: Determinar os patógenos que mais causam infecção em pacientes queimados e quantificar a frequência de cada um desses germes. Metodologia: revisão integrativa de literatura de trabalhos publicados de 2016 a 2021 , disponíveis nas bases de dados LILACS, PubMed e SciELO, foram incluídos 18 artigos no estudo. Resultados: A frequência de bactérias gram-positivas variou de 21,8 a 24\%, enquanto que de gram-negativas foi 70,3-78\%. Nas culturas de ferida detectou-se Pseudomonas (14,3-45,2\%), Staphylococcus (0-43,9\%), Acinetobacter (3,6-52,8\%), Proteus (0-16\%), Enterobacter (0-12,1\%), Klebsiella (0-28\%) e E. coli. (0-8,5\%). Já nas culturas de sítios diversos, Pseudomonas (17-49,9\%), Staphylococcus (6,5-31,2\%), Staphylococcus Coagulase Negativo (CoNS) (0-22\%), Acinetobacter (0-16,7\%), Proteus (0-0,9\%), Enterobacter (0-37\%), Klebsiella (0-14,3\%), Enterococo (0-2,8\%) e Serratia (0-1,2\%). Considerações Finais: Os agentes causadores de infecção identificados sofreram grandes variações 
de frequência na nossa análise. O conhecimento destes germes é fundamental para o correto manejo e redução da taxa de mortalidade dos pacientes queimados. Estudos desse tipo devem ser periodicamente realizados, para que não haja defasagem das informações ao longo do tempo.

Palavras-chave: Unidades de queimados; Queimaduras; Infecções.

\begin{abstract}
Objective: To determine the pathogens that most cause infection in burn patients and quantify their frequency. Methodology: An integrative literature review of articles published from 2016 to 2021, available in the databases LILACS, PubMed and SciELO, 18 articles were included in this study. Results: The frequency of gram-positive bacteria ranged from 21,8 to $24 \%$, while gram-negative $70,3-78 \%$. The wound cultures revealed Pseudomonas (14,3$45,2 \%)$, Staphylococcus (0-43,9\%), Acinetobacter (3,6-52,8\%), Proteus (0-16\%), Enterobacter (0-12,1\%), Klebsiella $(0-28 \%)$ and E. coli. (0-8,5\%). The general cultures tissues showed Pseudomonas (17-49,9\%), Staphylococcus (6.5$31.2 \%$ ), Coagulase Negative Staphylococcus (CoNS) (0-22\%), Acinetobacter (0-16,7\%), Proteus (0-0,9\%), Enterobacter (0-37\%), Klebsiella (0-14,3\%), Enterococci (0-2,8\%) and Serratia (0-1,2\%). Final Considerations: The detected agents of infection suffered several frequency variations in our analysis. The knowledge about these germs is essential for the correct management and reduction of the mortality rate in burn patients This kind of study must be carried out periodically, with the purpose of information does not lag over time.
\end{abstract}

Keywords: Burn units; Burns; Infections.

\title{
Resumen
}

Objetivo: Determinar los patógenos que más causan infección en pacientes quemados y cuantificar su frecuencia. Metodología: revisión de la literatura de trabajos publicados entre 2016 y 2021, disponibles en las bases de datos LILACS, PubMed y SciELO, se incluyeron 18 artículos en el estudio. Resultados: La frecuencia de bacterias grampositivas osciló entre $21,8 \%$ y $24 \%$, mientras que la de gramnegativas fue del $70,3 \%$ al $78 \%$. En cultivos de heridas mostraron Pseudomonas (14,3-45,2\%), Staphylococcus (0-43,9\%), Acinetobacter (3,6-52,8\%), Proteus (0$16 \%)$, Enterobacter (0-12,1\%), Klebsiella (0-28\%) y E. coli. (0-8,5\%). En cultivos de diferentes sitios, Pseudomonas (17-49,9\%), Staphylococcus (6,5-31,2\%), Staphylococcus coagulase negativo (CoNS) (0-22\%), Acinetobacter (0$16,7 \%)$, Proteus (0-0,9\%), Enterobacter (0-37\%), Klebsiella (0-14,3\%), Enterococci (0-2,8\%) y Serratia (0-1,2\%). Consideraciones finales: Los agentes causantes de infección identificados sufrieron amplias variaciones en la frecuencia en nuestro análisis. El conocimiento de estos gérmenes es fundamental para el correcto manejo y reducción de la tasa de mortalidad de los pacientes quemados. Los estudios de este tipo deben realizarse periódicamente, para que la información no se retrase en el tiempo.

Palabras clave: Unidades de quemados; Quemaduras; Infecciones.

\section{Introdução}

Dentre as várias definições, queimaduras são lesões teciduais orgânicas, com impacto primariamente epitelial, causadas por agentes externos. Elas podem ser classificadas quanto à sua etiologia, extensão, profundidade, região do corpo atingida e tempo de exposição (Cavalcante et al., 2021; Mélega, Viterbo \& Mendes, 2011; Souza et al., 2021).

Quanto à etiologia, podem ser divididas em queimadura térmica, elétrica, química, por radiação ionizante, e até mesmo frias. $\mathrm{O}$ agente causal mais frequente é o escaldo, seguido pela queimadura por chamas. Outros agentes importantes são flash burn (explosões), queimaduras de contato, eletricidade e produtos químicos ácidos ou álcalis. Os diferentes agentes causam padrões diferentes de lesões, desse modo, entender como a queimadura foi gerada é fundamental para a compreensão da resposta fisiopatológica induzida pela mesma e para o manejo adequado do paciente (Cavalcante et al., 2021; Mélega, Viterbo \& Mendes, 2011; Souza et al., 2021).

Quanto à profundidade, as queimaduras são divididas em primeiro, segundo e terceiro grau. As queimaduras de primeiro e segundo grau são consideradas de espessura parcial. Na de primeiro grau, a lesão é mais superficial e acomete apenas a epiderme. É caracterizada pelo eritema local, devido à vasodilatação dos capilares, e dor, porém não apresenta de bolhas nem repercussões hemodinâmicas. As queimaduras de segundo grau acometem a epiderme e a derme, gerando bolhas na pele atingida. São ainda subdivididas em superficiais e profundas. A superficial gera lesão até a camada papilar da derme e é caracterizada por coloração rósea abaixo das bolhas, aspecto úmido e dor intensa. A profunda atinge também a camada reticular da derme e alguns anexos cutâneos, tem aspecto mais pálido, mas é pouco dolorosa. As queimaduras de terceiro grau são as que acometem a pele em sua espessura total, incluindo nervos, glândulas e capilares sanguíneos, pode também lesar 
músculos e ossos. São as queimaduras mais graves, com grande potencial deformante. Tem característica variável, desde esbranquiçada até amarronzada, ou negro carbonizado. Costuma ser indolor devido à destruição das fibras nervosas. Esse tipo de lesão geralmente necessita de enxertia de pele pois há perda das funções de reepitelização local (Silva, Tavares, Tavares \& Santos, 2020; Mélega et al., 2011; Souza et al., 2021).

A extensão da queimadura é dada pela Área Corporal Queimada (ACQ). Pode ser estimada, de maneira mais precisa, pelo método de Lund-Browder ou, de maneira mais prática, pela regra dos nove (Figura 1), que sofre variações de acordo com a idade do paciente avaliado. Nesta última, o corpo do adulto é dividido em regiões que equivalem a múltiplos de $9 \%$ da superfície corporal total. A partir da ACQ, pode-se classificar o paciente em pequeno, médio e grande queimado. Crianças com SCQ inferior a $10 \%$ e os adultos com ACQ de $15 \%$ são considerados pequenos queimados. Se a ACQ estiver entre 10 e $20 \%$, considera-se médio queimado. O paciente é classificado como grande queimado quando a ACQ é maior que 10\% para crianças e 20\% para adultos, tendo em vista que queimaduras de primeiro grau são excluídas, pois não trazem repercussões sistêmicas ou funcionais (Silva et al., 2020; Mélega et al., 2011; Souza et al., 2021).

Figura 1 - Regra dos nove para estimativa da ACQ.

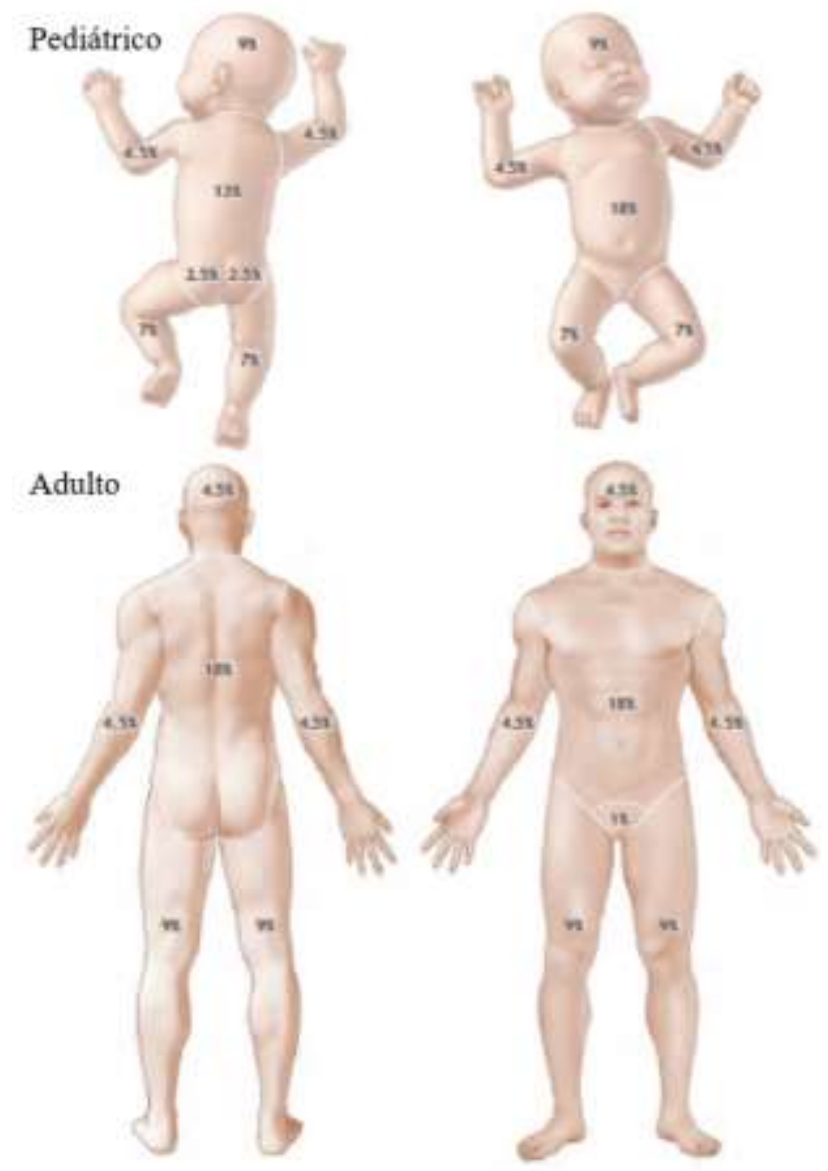

Fonte: ATLS (2018) (nossa tradução).

A fisiopatologia da queimadura é dividida por suas respostas locais e sistêmicas. Localmente, a queimadura gera três zonas bem definidas. A zona mais central, chamada de zona de coagulação, é o ponto da queimadura de maior dano, a lesão nesta zona é irreversível. Circundando a zona de coagulação, está a zona de estase ou zona de isquemia, devido ao comprometimento da vascularização nessa região. Esse tecido é potencialmente recuperável, porém danos adicionais como hipoperfusão, hipóxia e infecções podem tornar essa área necrótica. A zona mais externa, chamada de zona de hiperemia, é rica em células inflamatórias e promove a liberação de inúmeros mediadores tais como citocinas, cininas, histamina, tromboxane e 
radicais livres, promovendo aumento da perfusão tecidual local. Em queimaduras extensas, com mais de 10\% da ACQ, esses mediadores inflamatórios podem desencadear efeitos sistêmicos cardiovasculares, respiratórios, metabólicos e imunológicos (Mélega et al., 2011; Souza et al., 2021).

A pele íntegra serve de barreira protetora do corpo contra o ambiente externo, incluindo microrganismos capazes de gerar infecção. As queimaduras além de destruírem esse mecanismo de escudo, afetam sua função imunológica, possibilitando a rápida proliferação bacteriana, de modo que em um dia completo, pode crescer exponencialmente (Gonella, Quevedo \& Mendes, 2014). A queimadura leva a granulocitopenia, redução de citocinas inflamatórias, de interleucinas (IL) e de fatores de crescimento como IL-1, -2,-6-8 e-13, fator de necrose tumoral $\alpha$ (TNF- $\alpha$ ) e fator de crescimento endotelial vascular, causando um estado de imunossupressão (Mota et al., 2014). Além disso, pacientes com queimaduras graves, comumente necessitam de tempo prolongado de hospitalização, cateteres urinários, arteriais, acesso venoso central, intubação orotraqueal e/ou outros procedimentos que também aumentam o risco de contaminação. Somado a isso, a terapia antimicrobiana de amplo espectro é um fator importante para o desenvolvimento de infecções por agentes resistentes (El Hamzaoui, Barguigua, Larouz \& Maouloua, 2020; Lima, Oliveira, Araújo \& Oliveira, 2016).

As infecções em pacientes queimados devem ser consideradas de extrema importância pois podem levar ao desenvolvimento de condições ameaçadoras da vida, tais como bacteremia, sepse e falência de múltiplos órgãos, com grande contribuição para aumento da morbimortalidade e de custos dos cuidados de saúde dos pacientes queimados. Também é a causa mais comum de morte após lesão por queimadura, sendo que, cerca de três quartos das mortes nesses pacientes ocorrem devido à infecção (Lima, Oliveira, Araújo \& Oliveira, 2014; Ramakrishnan, Putli Bai, \& Babu, 2016).

Os fatores de risco para o desenvolvimento de infecção no paciente queimado são diversos, desde os relacionados ao próprio paciente, tais como extensão e profundidade da queimadura, idade, doenças preexistentes, desnutrição, temperatura e umidade da lesão, choque e acidose. Até os relacionados ao microrganismo causador da infecção: virulência, mobilidade, resistência bacteriana e produção de enzimas ou exotoxinas. As origens desses microrganismos também são diversas: do próprio acidente, da pele íntegra ao redor da lesão, da orofaringe, do reto e de outros meios endógenos. Podem também ser provenientes de terapias invasivas, translocação bacteriana, ou originados à distância. Também é possível a contaminação exógena, a partir da equipe, visitas e as infecções cruzadas. Dois recursos são amplamente utilizados para a análise qualitativa dessas bactérias, que são a técnica com coleta por uso de swab, e a biópsia de pele. Com sensibilidade de 100\%, o swab é útil na exclusão de infecção quando o resultado é negativo. Porém, há possibilidade de falsos-positivos em decorrência de sua especificidade de $77,4 \%$ (Gonella et al., 2014).

Os principais microrganismos causadores de infecção e sepse em pacientes queimados previamente elencados na literatura são Staphylococcus aureus, Staphylococcus coagulase negativo, Pseudomonas aeruginosa, Klebsiella sp, Enterobacter sp, Acinetobacter sp, Escherichia coli e Enterococcus faecalis. Já entre os fungos, destacam-se Candida albicans e Aspergillus sp. O controle das principais infecções no centro de queimados diminui o risco de complicações como a sepse. Fatores como a vigilância infecciosa, diagnóstico rápido e antibioticoterapia direcionada podem reduzir a taxa de mortalidade nesses pacientes (Sala, Lima, Simioni, \& Ugrinovich, 2016; Rempel, Tizzot \& Vasco, 2011). Sendo assim, o presente estudo tem como objetivo determinar esses patógenos e quantificar a frequência de cada um deles.

\section{Metodologia}

Foi feita uma revisão integrativa de literatura acerca dos principais microrganismos causadores de infecção em pacientes queimados e suas respectivas frequências. Tendo em vista o tipo de estudo realizado, não foi necessário aprovação de comitê de ética para a realização do mesmo. 
Com o tema central e o objetivo definidos, determinaram-se os termos de interesse da pesquisa: "unidade de queimados", "queimadura", "infecção" e "sepse". A partir deles, foi possível identificar os descritores controlados na base Descritores em Ciências da Saúde (DeCS/MeSH). Em português, foram definidos respectivamente "Unidades de Queimados", "Queimaduras", "Infecções" e "Sepse". Já em Inglês, definiram-se respectivamente "Burn Units", "Burns", "Infections" e "Sepsis". Para a coerência da pesquisa, os descritores foram organizados com os operadores booleanos "OR" e "AND" de acordo com o Quadro 1.

Utilizaram-se as bases de dados Literatura Latino-Americana e do Caribe em Ciências da Saúde (LILACS), Scientific Eletronic Library Online (SciELO) e National Library of Medicine (PubMed). Foram incluídos os estudos transversais, prospectivos ou retrospectivos, observacionais, analíticos, descritivos ou quantitativos publicados entre 2016 e 2021, nos idiomas português, inglês e espanhol. Foram excluídos deste estudo os trabalhos de revisão de literatura ou relatos de caso, artigos não disponíveis gratuitamente, incompletos, duplicatas e os que não tinham relação com o tema proposto. Para a consulta no LILACS foram usados os descritores em português supracitados e foram adicionados nos termos da pesquisa os anos de publicação de interesse (entre 2016 e 2021). Já para consulta no PubMed e SciELO, usaram-se os descritores em inglês, e os anos de publicação foram filtrados com as ferramentas disponibilizadas nas próprias bases de dados.

Quadro 1 - Estratégia de pesquisa em cada base de dados e seu quantitativo de resultados.

\begin{tabular}{|l|l|c|}
\hline \multicolumn{1}{|c|}{ Base de Dados } & \multicolumn{1}{|c|}{ Conteúdo da pesquisa } & Total de artigos encontrados \\
\hline LILACS & $\begin{array}{l}\text { (Unidades de Queimados) OR (Queimaduras) [Palavras] AND } \\
\text { (Infecções) OR (Sepse) [Palavras] AND ("2016" OR "2017" OR }\end{array}$ & 34 \\
& "2018" OR "2019" OR "2020" OR "2021") [País, ano de publicação] & \\
\hline PubMed & (burn units OR burns) AND (infections OR sepsis) & 131 \\
\hline SciELO & (burn units OR burns) AND (infections OR sepsis) & 06 \\
\hline
\end{tabular}

Fonte: Autores.

As pesquisas nas bases de dados resultaram em 34 artigos no LILACS, 131 no PubMed e 06 no SciELO. Esses trabalhos passaram por uma análise por seus títulos e resumo, levando em conta sua relação com o tema levantado, sobrando 36 artigos potenciais. Finalmente, após a leitura na íntegra desses 36 artigos, 18 foram incluídos neste trabalho, de acordo com o organograma (Figura 2).

Figura 2 - Organograma da seleção dos artigos da revisão.

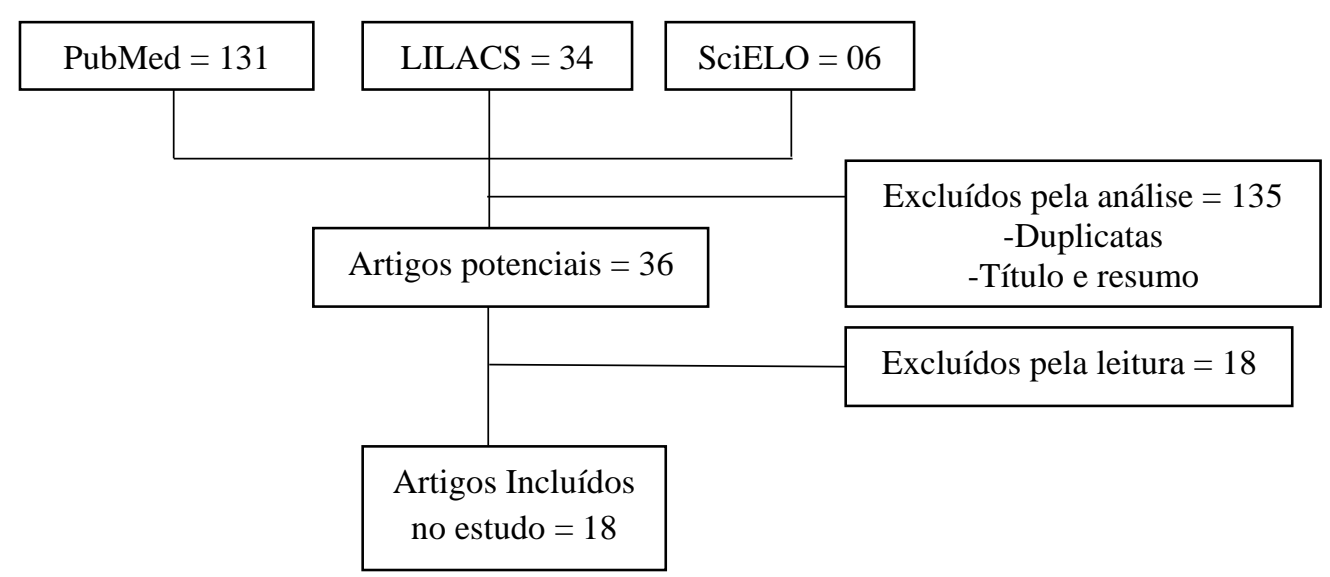

Fonte: Autores. 


\section{Resultados}

Dos 131 artigos resultantes do PubMed, 24 foram considerados potenciais para esta revisão, enquanto que no LILACS foram selecionados 10 dos 34 encontrados. Já do SciELO, foram selecionados 02 dos 06 encontrados na pesquisa. Desse modo, foram eleitos 36 artigos potenciais. Essa primeira seleção foi feita com base no título dos artigos e em seus resumos, buscando neles palavras-chaves como "queimados" e "infecção" ou equivalentes. Além disso, foram levados em conta os critérios de inclusão e exclusão, citados na metodologia.

A leitura completa dos 36 artigos potenciais possibilitou a escolha dos artigos incluídos nesse estudo. Foram selecionados os trabalhos com dados quantitativos da frequência dos agentes causadores de infecção e sepse em pacientes queimados a partir de culturas de quaisquer sítios. Desse modo, foram incluídos 14 artigos do PubMed, 04 do LILACS e nenhum do SciELO, totalizando 18 trabalhos. Todos os 14 artigos do PubMed estão na língua inglesa, enquanto que, dos artigos selecionados do LILACS, 02 estão em português e 02 estão em espanhol. A metodologia e os resultados resumidos desses artigos estão expostos no Quadro 2.

Quadro 2 - Resumo da metodologia e dos resultados dos artigos incluídos na revisão.

\begin{tabular}{|c|c|c|}
\hline $\begin{array}{c}\text { Primeiro } \\
\text { Autor (ano) }\end{array}$ & Resumo da Metodologia & Resumo dos resultados \\
\hline $\begin{array}{l}\text { Araújo } \\
(2020)\end{array}$ & $\begin{array}{l}\text { Estudo retrospectivo, transversal, por meio de análise de } \\
\text { todos os exames microbiológicos dos pacientes internados } \\
\text { por queimaduras na Unidade de Queimaduras do Hospital } \\
\text { Geral "José Pangella" de Vila Penteado, localizado na } \\
\text { cidade de São Paulo, durante o período de janeiro de } 2011 \\
\text { até o final de dezembro de } 2018 \text {. Foram avaliados } 426 \\
\text { exames microbiológicos de } 250 \text { pacientes diferentes. }\end{array}$ & $\begin{array}{l}\text { Dos } 426 \text { exames analisados, foram isolados } 495 \\
\text { microrganismos, sendo } 436 \text { bactérias }(88,080 \%) \text { e } 59 \text { fungos } \\
(11,919 \%) . \\
\text { Staphylococcus sp. }(130 \text { casos ou } 26,262 \%) ; \\
\text { Peudomonas sp. }(102 \text { casos ou } 20,606 \%) ; \\
\text { Klebsiella sp. }(61 \text { casos ou } 12,323 \%) ; \\
\text { Candida sp. }(58 \text { casos ou } 11,717 \%) ; \\
\text { Acinetobacter sp. }(57 \text { casos ou } 11,515 \%) .\end{array}$ \\
\hline Asati (2017) & $\begin{array}{l}\text { Um estudo prospectivo foi realizado de março a agosto de } \\
2015 \text { em } 133 \text { amostras de pus de pacientes queimados } \\
\text { recebidos no Departamento of Microbiology, Pt. B.D. } \\
\text { Sharma PGIMS, Rohtak, Haryana, Índia. }\end{array}$ & $\begin{array}{l}\text { Destes, } 82 \quad(61,6 \%) \text { infecção monomicrobiana e } \\
\text { multimicrobiana em } 51(38,4 \%) \text { amostras. Bactérias gram- } \\
\text { negativas em } 76 \% \text { e gram-positivos em } 24 \% \text {. } \\
\text { P. aeruginsa }(32,1 \%) ; \\
\text { Klebsiella spp. }(16,3 \%) ; \\
\text { Staphylococcus aureus }(14,7 \%) \text {. }\end{array}$ \\
\hline $\begin{array}{l}\text { Balmelli } \\
\text { (2018) }\end{array}$ & $\begin{array}{l}\text { Estudo descritivo, observacional, restrospectivo de corte } \\
\text { transversal. Incluídos todos os pacientes queimados no } \\
\text { serviço de pediatria do Centro nacional de queimados e } \\
\text { cirurgias reconstrutivas (CENQUER) de janeiro de } 2017 \text { até } \\
2018 \text {, com um total de } 220 \text { pacientes. }\end{array}$ & $\begin{array}{l}\text { Foi documentado um total de } 128(58 \%) \text { pacientes que } \\
\text { evoluíram com infecção, desses, } 29(23 \%) \text { evoluíram com } \\
\text { infecção da ferida. } \\
\text { P. aeruginosa MRR } 31 \%(\mathrm{n}=9) \text {; } \\
\text { Acinetobacter } \operatorname{spp} \text { MRR } 17 \%(\mathrm{n}=5) \text {; } \\
\text { MRSA } 13 \%(\mathrm{n}=4) \text {; } \\
\text { MSSA } 6 \%(\mathrm{n}=2) \text {; } \\
\text { Klebsiella spp } 6 \%(\mathrm{n}=2) \text {. }\end{array}$ \\
\hline $\begin{array}{l}\text { El } \\
\text { Hamzaoui } \\
(\mathbf{2 0 2 0})\end{array}$ & $\begin{array}{l}\text { Estudo realizado no Serviço de Queimaduras do Hospital } \\
\text { Mohammed V, Cidade de Meknes, Marrocos, de janeiro de } \\
2015 \text { a janeiro de 2016, } 126 \text { pacientes foram internados. }\end{array}$ & $\begin{array}{l}86(68,25 \%) \text { de } 126 \text { pacientes foram diagnosticado com } \\
\text { infecção: } \\
\text { Staphylococcus aureus }(33,85 \%) ; \\
\text { Pseudomonas spp. }(18,46 \%) ; \\
\text { Acinetobacter baumannii }(15,38 \%) ; \\
\text { Klebsiella pneumoniae }(13,85 \%) ; \\
\text { Escherichia coli }(8,46 \%) ; \\
\text { Proteus mirabilis }(4,42 \%) \text {. }\end{array}$ \\
\hline $\begin{array}{l}\text { Emami } \\
(2020)\end{array}$ & $\begin{array}{l}\text { Estudo transversal realizado em pacientes queimados } \\
\text { hospitalizados no hospital afiliado ao hospital Amir-Al- } \\
\text { Momenin com a Universidade de Ciências Médicas de } \\
\text { Shiraz, Shiraz, Irã (SUMS). Durante um período de três } \\
\text { anos, de janeiro de } 2016 \text { a dezembro de } 2018 \text {. Um total de } \\
3420 \text { amostras de } 615 \text { pacientes hospitalizados. }\end{array}$ & $\begin{array}{l}960(28 \%) \text { amostras de crescimento positivo foram coletados } \\
\text { de } 615 \text { indivíduos queimados, infecção da ferida foi a maior } \\
\text { (264 / Incidência de } 960,27,5 \%) \text {. } \\
\text { P. aeruginosa }(49,9 \%) ; \\
\text { Klebsiellasp. }(9,7 \%) ; \\
\text { Acinetobacter sp. }(7,2 \%) \text {; }\end{array}$ \\
\hline
\end{tabular}




\begin{tabular}{|c|c|c|}
\hline & & S. aureus $(6,5 \%)$. \\
\hline $\begin{array}{l}\text { Forson } \\
\text { (2017) }\end{array}$ & $\begin{array}{l}\text { Estudo transversal realizado no Centro Nacional de Cirurgia } \\
\text { Plástica Reconstrutiva e Queimaduras (NRPSBC) do } \\
\text { Hospital Universitário Korle-Bu (KBTH), em Gana, de abril } \\
\text { a julho de } 2016 \text {, com um total de } 50 \text { pacientes. }\end{array}$ & $\begin{array}{l}50 \text { amostras cultivadas, } 43(86 \%) \text { foram positivas para } \\
\text { bactérias, } 7 \text { ( } 42 \%) \text { não apresentaram crescimento. } \\
\text { Pseudomonas sp. }(30,2 \%) ; \\
\text { Acinetobacter }(20,9 \%) ; \\
\text { Proteus mirabillis }(16,3 \%) ; \\
\text { Enterobacter } \text { sp. }(11,6 \%) ; \\
\text { Klebsiella } \text { sp. }(7,0 \%) ; \\
\text { Citrobacter } \text { sp. }(4,7 \%) ; \\
\text { Klebsiella oxytoca }(4,7 \%) ; \\
\text { Proteus vulgaris }(2,3 \%) ; \\
\text { Staphylococcus aureus }(2,3 \%) .\end{array}$ \\
\hline $\begin{array}{l}\text { Güldoğan } \\
\text { (2017) }\end{array}$ & $\begin{array}{l}\text { Estudo retrospectivo que analisou prontuários dos pacientes } \\
\text { internados em um Centro de Tratamento de Queimados entre } \\
01 / 01 / 2014 \text { e } 31 / 07 / 2015 \text { de um hospital de referência da } \\
\text { Turquia. Foram } 349 \text { pacientes incluídos no estudo. }\end{array}$ & $\begin{array}{l}\text { Nas } 31 \text { culturas da ferida foram detectados: } \\
\text { Acinetobacter baumannii } 36,1 \%(\mathrm{n}=13) ; \\
\text { Pseudomonas aeruginosa } 22,2 \%(\mathrm{n}=8) ; \\
\text { P. aeroginosa }+ \text { A. baumannii } 13,9 \%(\mathrm{n}=4) \text {; } \\
\text { MRSA } 2,8 \%(\mathrm{n}=1) \text {; } \\
\text { C. albicans } 2,8 \%(\mathrm{n}=1) \text {. }\end{array}$ \\
\hline $\begin{array}{l}\text { Gupta } \\
(2019)\end{array}$ & $\begin{array}{l}\text { Estudo retrospectivo de pacientes internados na Unidade de } \\
\text { Tratamento de Queimados de um hospital terciário, } \\
\text { Jharkhand, Índia, durante janeiro de } 2015 \text { a julho } 2017 \text { foi } \\
\text { realizado. Amostras de } 299 \text { pacientes foram analisadas no } \\
\text { período. }\end{array}$ & $\begin{array}{l}299 \text { swabs de feridas de pacientes queimados, } 61,87 \% \text { (n } \\
\text { 185) positivas para infecção bacteriana: } \\
\text { Pseudomonas spp ( } 43 \%) ; \\
\text { Klebsiella pneumoniae }(28 \%) ; \\
\text { Acinetobacter baumannii }(14,83 \%) ; \\
\text { E. coli }(6,59 \%) \text {; } \\
\text { Enterobacter cloacae }(2,20 \%) ; \\
\text { Burkholderia cepacia }(1,65 \%) ; \\
\text { Staphylococcus aureus }(1,62 \%) ; \\
\text { Proteus mirabilis }(1,10 \%) .\end{array}$ \\
\hline $\begin{array}{l}\text { Horta } \\
(\mathbf{2 0 2 0})\end{array}$ & $\begin{array}{l}\text { Estudo transversal, retrospectivo, sobre infecções fúngicas } \\
\text { em pacientes queimados internado no Centro Hospitalar São } \\
\text { João (CHSJ) Queimados na UTI durante os anos 2013-2015. } \\
178 \text { pacientes foram admitidos, destes, } 172 \text { pacientes foram } \\
\text { selecionados para este estudo. }\end{array}$ & $\begin{array}{l}\text { Trinta e oito pacientes }(22,1 \%) \text { tiveram um fungo positivo na } \\
\text { cultura: } \\
\text { Candida spp. ( } 20 \text { pacientes - } 52,6 \%) \text {; } \\
\text { Candida albicans }(15 \text { pacientes }-39,5 \%) \text {; } \\
\text { Aspergillus spp. }(1 \text { paciente }-2,6 \%) \text {; } \\
\text { Não identificados ( } 2 \text { casos }-5,3 \%) \text {. }\end{array}$ \\
\hline $\begin{array}{l}\text { Latifi } \\
\text { (2017) }\end{array}$ & $\begin{array}{l}\text { Foram coletados prospectivamente os dados de pacientes } \\
\text { queimados do programa de registro de gravação do país, e } \\
\text { inseriu-se esses dados em um questionário especial, } \\
\text { alcançando um total de } 1.721 \text { pacientes analisados. }\end{array}$ & $\begin{array}{l}47,7 \% \text { dos pacientes realizaram biópsia da ferida, } 481 \\
(38,54 \%) \text { deles com cultura positiva: } \\
\text { Staphylococcus spp. }(55,1 \%) \\
\text { Pseudomonas aeruginosa }(14,29 \%) \text {; } \\
\text { Enterococcus }(12,24 \%) ; \\
\text { E. coli }(4 \%) \text {; } \\
\text { Klebsiella e Proteus (ambos } 2 \%) \text {. }\end{array}$ \\
\hline Lima (2016) & $\begin{array}{l}\text { Estudo prospectivo, observacional, desenvolvido de janeiro a } \\
\text { dezembro de 2012, na Unidade de Tratamento de } \\
\text { Queimados do Hospital João XXIII (UTQ-HJXIII) da } \\
\text { Fundação Hospitalar do Estado de Minas Gerais, em Belo } \\
\text { Horizonte, MG, com } 136 \text { pacientes de } 0 \text { a } 20 \text { anos. }\end{array}$ & $\begin{array}{l}68 \text { culturas, } 31(22,8 \%) \text { positivas, sendo } 12 \text { pacientes com } \\
\text { dois ou mais agentes isolados: } \\
\text { Staphylococcus aureus }(31,2 \%) \text {; } \\
\text { Pseudomonas aeruginosa }(27,1 \%) \text {; } \\
\text { Acinetobacter baumanni }(16,7 \%) \text {. }\end{array}$ \\
\hline $\begin{array}{l}\text { Mater } \\
(\mathbf{2 0 2 0})\end{array}$ & $\begin{array}{l}\text { Revisão retrospectiva de prontuários de todos os pacientes } \\
\text { queimados internados Al-Noor Specialist Hospital, Riyadh, } \\
\text { Arábia Saudita, entre janeiro de } 2016 \text { e janeiro de } 2017 \text {. No } \\
\text { total, } 250 \text { pacientes queimados foram incluídos. }\end{array}$ & $\begin{array}{l}83 \text { swabs de ferida, sendo } 48(57,8 \%) \text { positivas: } \\
\text { MRSA }(36,4 \%) \text {; } \\
\text { Pseudomonas aeruginosa }(22,7 \%) \text {; } \\
\text { Enterobacter cloacae }(12,12 \%) \text {; } \\
\text { A. baumannii, MSSA e K. pneumoniae }(7,58 \%) \text {. }\end{array}$ \\
\hline $\begin{array}{l}\text { Ramakrish } \\
\text { nan } \\
(2016)\end{array}$ & $\begin{array}{l}\text { Estudo com } 30 \text { esfregaços de feridas de queimaduras obtidos } \\
\text { a partir de } 14 \text { pacientes pediátricos queimados entre } \\
\text { novembro de } 2013 \text { e março de } 2014 \text {. }\end{array}$ & $\begin{array}{l}\text { Pseudomonas aeruginosa }(33,3 \%) \\
\text { Acinetobacter }(23,3 \%) \\
\text { Staphylococcus aureus }(16,6 \%)\end{array}$ \\
\hline
\end{tabular}




\begin{tabular}{|c|c|c|}
\hline $\begin{array}{l}\text { Roham } \\
\text { (2017) }\end{array}$ & $\begin{array}{l}\text { Revisão retrospectiva de um estudo prospectivo do banco de } \\
\text { dados coletado de queimaduras em adultos e crianças } \\
\text { pacientes internados no Hospital de Queimados de Motahari } \\
\text { em Teerã, que tinham um cateter venoso central em um ano. } \\
\text { Um total de } 191 \text { pacientes queimados foram elegíveis para o } \\
\text { estudo. }\end{array}$ & $\begin{array}{l}\text { A positividade da cultura realizada variou a depender do } \\
\text { sítio investigado: em cateter femoral foi de } 32,93 \% \text {; nas } \\
\text { veias jugular interna e subclávia, combinadas, foi } \\
\text { ligeiramente superior, } 29,13 \% \text {. } \\
\text { As bactérias mais encontradas em cateter venoso central, } \\
\text { independentemente do sítio: Pseudomonas aeruginosa }(27,2- \\
50 \%) \text {, Staphylococcus spp. }(0-35 \%) \text {, Acinetobacter } \\
\text { baumanni }(0-35,9 \%) \text {, Klebsiella spp }(0-50 \%) \text {, Serratia }(0- \\
9 \%) \text {, Proteus }(0-4 \%) \text {. }\end{array}$ \\
\hline $\begin{array}{l}\text { Rosanova } \\
(2019)\end{array}$ & $\begin{array}{l}\text { Estudo retrospectivo e descritivo de pacientes pediátricos } \\
\text { queimados com osteomielite fúngica confirmada, internados } \\
\text { no Hospital Juan P. Garrahan, entre janeiro de } 2007 \text { até } \\
\text { janeiro de } 2017 \text {, com um total de } 600 \text { pacientes. }\end{array}$ & $\begin{array}{l}\text { Dos } 600 \text { pacientes queimados atendidos no período, } 9 \\
\text { apresentaram osteomielite fúngica confirmada }(1.5 \%) \text { : } \\
\text { Fusarium spp em } 03 \text { pacientes }(33,3 \%) ; \\
\text { Mucor spp, um paciente }(11,1 \%) ; \\
\text { Trichosporon asahii, um paciente }(11,1 \%) ; \\
\text { Candida albicans, dois pacientes }(22,2 \%) ; \\
\text { Candida parapsilosis, dois pacientes }(22,2 \%) \text {. }\end{array}$ \\
\hline $\begin{array}{l}\text { van Duin } \\
(2017)\end{array}$ & $\begin{array}{l}\text { Estudo retrospectivo, pacientes internados na unidade de } \\
\text { queimados de um grande centro terciário de referência em } \\
\text { queimados entre } 01 / 01 / 2004 \text { e } 31 / 12 / 2013 \text { foram incluídos. } \\
\text { Nesse período, } 5.524 \text { pacientes adultos com queimaduras } \\
\text { foram incluídos neste estudo. }\end{array}$ & $\begin{array}{l}\text { As cinco classes de patógenos mais comuns foram: } \\
\text { Enterobacteriaceae }(37 \%) \text {; } \\
\text { Staphylococcus aureus }(26 \%) \text {; } \\
\text { Staphyloccocci coagulase-negativa }(22 \%) \text {; } \\
\text { Candida spp. }(19 \%) ; \\
\text { Pseudomonas spp. }(17 \%) \text {. }\end{array}$ \\
\hline Vaez (2016) & $\begin{array}{l}\text { Estudo transversal entre março de } 2013 \text { e abril de } 2014 \text { em } \\
\text { um hospital de referência para queimados na província de } \\
\text { Isfahan, Irã. } 250 \text { swabs de feridas coletados de pacientes } \\
\text { queimados internados no hospital foram avaliados. }\end{array}$ & $\begin{array}{l}\text { Dos } 250 \text { esfregaços de feridas, } 78(31 \%) \text { amostras positivas. } \\
\text { Destas, } 17(21,8 \%) \text { eram Gram-positivas e } 61(78,2 \%) \text { eram } \\
\text { Gram-negativas. } \\
\text { Pseudomonas aeruginosa }(\mathrm{n}=38 ; 48,7 \%) \text {; } \\
\text { Staphylococcus aureus }(\mathrm{n}=17 ; 21,8 \%) ; \\
\text { Acinetobacter baumannii }(\mathrm{n}=9 ; 11,5 \%) ; \\
\text { Klebsiella } \text { spp. }(\mathrm{n}=8 ; 10,3 \%) ; \\
\text { E. coli }(\mathrm{n}=3 ; 3,8 \%) ; \\
\text { Serratia } \text { spp. }(\mathrm{n}=3 ; 3,8 \%) \text {. }\end{array}$ \\
\hline $\begin{array}{l}\text { Wardhana } \\
\text { (2017) }\end{array}$ & $\begin{array}{l}\text { Estudo retrospectivo descritivo que revisou os dados de } \\
\text { pacientes queimados internados na Unidade de Queimados } \\
\text { do RSCM (Indonésia) entre setembro de } 2016 \text { e novembro } \\
\text { de } 2016 \text {. No total, foram avaliados testes de } 36 \text { pacientes. }\end{array}$ & $\begin{array}{l}\text { Das } 27 \text { culturas da ferida coletadas: Bactérias gram- } \\
\text { negativas } 70,3 \%(\mathrm{n}=19) \text {, Bactérias gram-positivas } 22,2 \% \\
(\mathrm{n}=6) \text { e } 7,4 \%(\mathrm{n}=2) \text { sem crescimento bacteriano. } \\
\text { Pseudomonas aeruginosa }(\mathrm{n}=7 ; 28 \%) ; \\
\text { Acinetobacter baumannii }(\mathrm{n}=5 ; 20 \%) ; \\
\text { Enterobacter aerogenes e } S . \text { epidermidis }(\mathrm{n}=3 ; 12 \%) ; \\
\text { Klebsiella pneumoniae e Proteus mirabilis }(\mathrm{n}=2 ; 8 \%) \text {, } \\
\text { Enterococcus faecalis, Bacillus sp. e MRSS }(\mathrm{n}=1 ; 4 \%) \text {. }\end{array}$ \\
\hline
\end{tabular}

Legenda: $\mathrm{n}$ - tamanho da amostra. \% - frequência relativa. MRSA - Staphylococcus Aureus Resistente à Meticilina. MSSA - Staphylococcus Aureus Sensível à Meticilina. MRR - Multirresistente. Fonte: Autores.

Dos 18 artigos selecionados, 16 trazem dados de infecção bacteriana de pacientes queimados. Destes, 11 disponibilizam o padrão de infecção a partir de culturas da ferida (Tabela 1), 04 trazem dados a partir de culturas de sítios variados (Tabela 2) e 01, Roham et al. (2011), fez um estudo baseado apenas em culturas de cateter venoso central.

Nos estudos selecionados, a frequência de bactérias gram-positivas variou de 21,8-24\%, enquanto que de gramnegativas 70,3-78\%. Levando em conta apenas as culturas de ferida do paciente queimados (Tabela 1), os principais gêneros de bactérias detectadas foram Pseudomonas (14,3-45,2\%), Staphylococcus (0-43,9\%), Acinetobacter (3,6-52,8\%), Proteus (0$16 \%)$, Enterobacter (0-12,1\%), Klebsiella (0-28\%), E. coli. (0-8,5\%). 
Tabela 1 - Frequência de bactérias mais isoladas em cultura de feridas.

\begin{tabular}{|c|c|c|c|c|c|c|c|c|}
\hline \multirow{2}{*}{$\begin{array}{l}\text { Primeiro } \\
\text { Autor } \\
\text { (ano) }\end{array}$} & \multirow[b]{2}{*}{$\mathbf{n}$} & \multicolumn{7}{|c|}{ Bactérias encontradas (\%) } \\
\hline & & Pseudomonas & Acinetobacter & Proteus & Enterobacter & Klebsiela & $\begin{array}{c}E . \\
\text { Coli }\end{array}$ & Staphylococcus \\
\hline $\begin{array}{l}\text { Asati } \\
(2017)\end{array}$ & 190 & 32,1 & 5,3 & 8,4 & 2,6 & 16,3 & 3,7 & 14,7 \\
\hline $\begin{array}{l}\text { Balmelli } \\
(2018)\end{array}$ & 29 & 31 & 17,2 & 0 & 3,4 & 6,9 & 0 & 20,7 \\
\hline $\begin{array}{l}\text { El hamzaoui } \\
(2020)\end{array}$ & 126 & 18,5 & 15,38 & 5,4 & 0 & 13,8 & 8,5 & 37,7 \\
\hline $\begin{array}{l}\text { Forson } \\
(2017)\end{array}$ & 50 & 26 & 18 & 16 & 10 & 10 & 0 & 0 \\
\hline $\begin{array}{l}\text { Güldoğan } \\
\text { (2017) }\end{array}$ & 36 & 38,9 & 52,8 & 0 & 0 & 0 & 2,8 & 5,6 \\
\hline $\begin{array}{l}\text { Gupta } \\
(2019)\end{array}$ & 299 & 45,2 & 14,8 & 1,1 & 2,2 & 28 & 6,6 & 1,62 \\
\hline $\begin{array}{l}\text { Latifi } \\
(2017)\end{array}$ & 821 & 14,3 & 0 & 2,0 & 0 & 2,0 & 4,1 & 2 \\
\hline $\begin{array}{l}\text { Mater } \\
(2020)\end{array}$ & 83 & 22,7 & 7,6 & 1,5 & 12,1 & 7,6 & 0 & 43,9 \\
\hline $\begin{array}{l}\text { Ramakrishnan } \\
\text { (2016) }\end{array}$ & 30 & 33,3 & 23,3 & 0 & 0 & 0 & 0 & 16,6 \\
\hline $\begin{array}{l}\text { Vaez } \\
(2016)\end{array}$ & 250 & 15,2 & 3,6 & 0 & 0 & 3,2 & 1,2 & 6,8 \\
\hline $\begin{array}{l}\text { Wardhana } \\
(2017)\end{array}$ & 27 & 25,9 & 18,5 & 7,4 & 11,1 & 7,4 & 0 & 14,8 \\
\hline
\end{tabular}

Legenda: $\mathrm{n}$ - tamanho da amostra. \% - frequência relativa. Fonte: Autores.

Nos trabalhos que não distinguiram os sítios de cultura realizadas (Tabela 2), a predominância foi de Pseudomonas (17-49,9\%), Staphylococcus (6,5-31,2\%), Staphylococcus Coagulase Negativo (CoNS) (0-22\%), Acinetobacter (0-16,7\%), Proteus (0-0,9\%), Enterobacter (0-37\%), Klebsiella (0-14,3\%), Enterococo (0-2,8\%) e Serratia (0-1,2\%).

Tabela 2 - Frequência de bactérias mais isoladas em culturas de sítios variados.

\begin{tabular}{|c|c|c|c|c|c|c|c|c|}
\hline \multirow{2}{*}{$\begin{array}{l}\text { Primeiro } \\
\text { Autor } \\
\text { (ano) }\end{array}$} & \multirow[b]{2}{*}{$\mathbf{n}$} & \multicolumn{7}{|c|}{ Bactérias encontradas (\%) } \\
\hline & & Pseudomonas & Acinetobacter & Enterobacter & Klebsiella & Staphylococcus & CoNS & Enterococo \\
\hline $\begin{array}{l}\text { Araújo } \\
(2020)\end{array}$ & 426 & 23,9 & 13,3 & 0 & 14,3 & 30,5 & 0 & 2,8 \\
\hline $\begin{array}{l}\text { Emami } \\
(2020)\end{array}$ & 960 & 49,9 & 7,2 & 0 & 9,7 & 6,5 & 0 & 0 \\
\hline $\begin{array}{l}\text { Lima } \\
(2016)\end{array}$ & 31 & 27,1 & 16,7 & 0 & 0 & 31,2 & 0 & 0 \\
\hline $\begin{array}{l}\text { van Duin } \\
(2016)\end{array}$ & 774 & 17 & 0 & 37 & 0 & 26 & 22 & 0 \\
\hline
\end{tabular}




\section{Discussão}

Há concordância dos resultados com o estudo de Rempel et al. (2011), onde o percentual médio de culturas positivas foi de 58,3\%. Ainda neste estudo, as espécies mais encontradas foram Pseudomonas aeruginosa (38,7\%), Acinetobacter baumannii (24,4\%) e Staphylococcus aureus (19,3\%). Já no estudo de Gragnani et al. (2014), com análise de 159 culturas, demonstrou-se taxa de positividade total de 34,5\%. Nas culturas de vigilância, notou-se maior prevalência de Staphylococcus coagulase-negativa, Escherichia coli e Staphylococcus aureus. Quando analisadas apenas as culturas positivas, os agentes mais frequentes foram Staphylococcus Coagulase-negativo (33\%), Pseudomonas aeruginosa (24\%), Acinetobacter spp. (22\%) e Klebsiella pneumoniae (5\%).

Güldoğan, Kendirci, Tikici, Gündoğdu, e Yastı, (2017) mostraram que a ferida foi o foco séptico mais frequentemente encontrado. Em 69,6\% pacientes, o foco se limitou à ferida de queimadura e o local da ferida contribuiu com a sepse em um total de $91,3 \%$ dos pacientes. Nesta revisão, o percentual dos pacientes queimados que desenvolveram sepse variou de 19,8\% a $63,9 \%$, considerando apenas os artigos que trouxeram esses dados. Entre os agentes causadores de sepse principais, encontramse Pseudomonas (13-43\%), Staphylococcus (0-27\%), Acinetobacter (11,1-41\%), Klebsiella spp (0-28,4\%), E. coli. (0-6\%), Serratia (0-4\%) e Enterobacter (0-6,7\%). Isso corrobora com o estudo de Mota et al. (2014), na Unidade de Queimados do Hospital das Clínicas da Universidade de São Paulo, onde foi observada uma prevalência de 55\% de infecções em pacientes queimados. Além disso, a infecção estava presente em até $75 \%$ dos casos dos pacientes queimados que foram a óbito, sendo considerada a maior responsável pela deterioração clínica fatal. A prevalência de sepse nesses pacientes variou de $8 \%$ a $65 \%$ e foi atribuída como causa de morte em $28 \%$ a $65 \%$ dos casos.

Nos trabalhos que analisaram hemoculturas, estas tiveram resultados positivos de 7,6 a 39\% nesses estudos. Com predomínio de Pseudomonas (51,6-100\%), Staphylococcus (7,7-40\%), Acinetobacter baumannii (0-50\%), Klebsiella spp (17,7-27\%), E. coli. (0-3\%), Proteus (0-10\%) e Enterococo (0-9,2\%) (Güldoğan et al., 2017, 2017; Mater et al., 2020; Balmelli, Sandoval, \& Canata, 2018).

O cateter venoso central teve cultura positiva em 12,3\% no estudo de Latifi, e Karimi (2017), que avaliou cateteres venosos de sítios diversos. Já segundo Roham et al. (2017), a positividade da cultura realizada variou a depender do sítio investigado, de modo que em cateter femoral foi de 32,93\%. Nas veias jugular interna e subclávia, combinadas, foi ligeiramente superior, 29,13\%. As bactérias mais encontradas em cateter venoso central, independentemente do sítio, foram Pseudomonas aeruginosa (27,2-50\%), Staphylococcus spp. (0-35\%), Acinetobacter baumanni (0-35,9\%), Klebsiella spp (050\%), Serratia (0-9\%), Proteus (0-4\%).

Com relação à presença de infecção fúngica em pacientes queimados (Tabela 3), Balmelli et al. (2018) conseguiu destacar os principais agentes por sítio. Nas culturas da ferida ( $\mathrm{n}=29)$, a predominância era de Fusarium $(17,2 \%$ - n=5), Aspergillus fumigatus $(17,2 \%$ - $\mathrm{n}=5)$, Alternaria spp e Candida tropicalis (10,3\% cada - $\mathrm{n}=3)$. Candida albicans, Aureobasidium pullulans, Dreschslera, Penicillium, Mucor, Rhodotorula e filamentos não tipificados foram identificados em uma amostra cada. Nas culturas de cateter $(\mathrm{n}=11)$, houve detecção de Candida albicans e Candida parapsilosis em 03 amostras cada (27,2\%) e Candida sp não C. albicans em 01 amostra (9\%). Nos casos de sepse associada à ferida da queimadura $(\mathrm{n}=48)$ os principais fungos identificados foram Candida albicans $(10,4 \%-\mathrm{n}=5)$, fungos filamentosos não tipificados (8,3\% - n=4), Candida sp não albicans $(6,2 \%$ - n=3), Candida guillermondi e Trichosporum asahi em duas amostras cada (4,2\%), Candida tropicalis, Candida lusitaniae, Candida glabrata e Mucor em uma amostra cada (2\%).

Horta, Tomaz, Egipto, e Silva (2020) realizaram um trabalho com 172 indivíduos de uma unidade de tratamento intensivo para pacientes queimados. Destes, 38 pacientes $(22,1 \%)$ tiveram ao menos um fungo positivo na cultura. A maioria dos fungos isolados foram obtidos a partir de amostras de partes moles (60,5\%), seguido por amostras urinárias (23,7\%) e, finalmente, amostras de hemoculturas e secreções respiratórias (7,9\% cada). Os germes mais comuns encontrados nessas 
amostras foram Candida spp. em 20 pacientes (52,6\%), seguido por Candida albicans (15 pacientes - 39,5\%), Aspergillus spp. (1 paciente -2,6\%) e em 2 casos (5,3\%) os agentes não foram identificados. Rosanova et al. (2019) realizou um estudo com 9 pacientes pediátricos que desenvolveram osteomielite fúngica. As culturas do tecido ósseo mostraram a predominância de Fusarium spp (3 casos -33,3\%), Candida parapsilosis ( 2 casos - 22,2\%), Candida albicans ( 2 casos $-22,2 \%)$, Trichosporon asahii e Mucor spp. (1 caso cada - 11,1\%).

Tabela 3 - Frequência de fungos mais isoladas em culturas de sítios diversos.

\begin{tabular}{|c|c|c|c|c|c|c|c|c|c|}
\hline \multirow[b]{2}{*}{$\begin{array}{l}\text { Primeiro } \\
\text { Autor (ano) }\end{array}$} & \multirow[b]{2}{*}{$\begin{array}{l}\text { Fonte da } \\
\text { cultura }\end{array}$} & \multirow[b]{2}{*}{$\mathbf{n}$} & \multicolumn{7}{|c|}{ Bactérias encontradas (\%) } \\
\hline & & & $\begin{array}{l}\text { Candida } \\
\text { spp }\end{array}$ & $\begin{array}{l}\text { Candida } \\
\text { albicans }\end{array}$ & $\begin{array}{c}\text { Candida } \\
\text { não } \\
\text { albican }\end{array}$ & Aspergillus & Fusarium & Trichosporon & $\begin{array}{l}\text { Mucor } \\
\text { spp. }\end{array}$ \\
\hline $\begin{array}{l}\text { Araujo } \\
(2020)\end{array}$ & Diversas & 426 & 13,6 & - & - & 0 & 0 & 0,9 & 0 \\
\hline $\begin{array}{l}\text { Balmelli } \\
(2018)\end{array}$ & Ferida & 29 & - & 3,4 & 10,3 & 17,2 & 17,2 & 0 & 3,4 \\
\hline $\begin{array}{l}\text { Horta } \\
(2020)\end{array}$ & Diversas & 38 & - & 39,5 & 52,6 & 2,6 & 0 & 0 & 0 \\
\hline $\begin{array}{l}\text { Güldoğan } \\
\text { (2017) }\end{array}$ & Ferida & 31 & - & 2,8 & 0 & 0 & 0 & 0 & 0 \\
\hline $\begin{array}{l}\text { Mater } \\
(2020)\end{array}$ & Ferida & 83 & - & 1,5 & 0 & 0 & 0 & 0 & 0 \\
\hline $\begin{array}{l}\text { Rosanova } \\
(2019)\end{array}$ & Osso & 9 & - & 22,2 & 22,2 & 0 & 33,3 & 11,1 & 11,1 \\
\hline $\begin{array}{l}\text { van Duin } \\
\text { (2016) }\end{array}$ & Diversas & 774 & 19 & - & - & 0 & 0 & 0 & 0 \\
\hline
\end{tabular}

Legenda: $\mathrm{n}$ - tamanho da amostra. \% - frequência relativa. Fonte: Autores.

Comparando os diversos trabalhos, chama atenção a grande variabilidade da predominância dos microrganismos a depender da instituição, superfície corporal queimada e tempo de internação.

A severidade da queimadura, avaliada pela Superfície Corporal Queimada (SCQ), segundo Mater et al. (2020), é diretamente proporcional à taxa de culturas positivas tanto na população pediátrica quanto na adulta. Tanto nas hemoculturas quanto nas culturas de feridas, os percentuais de positividade foram maiores à medida em que a SCQ foi $\geq 40 \%$ e foram substancialmente menores quando SCQ foi <40\%. Silva et al. (2020) corrobora que, as infecções tendem a acometer mais os pacientes com SCQ maior que 30\%. Sendo assim, a equipe multidisciplinar deve atentar-se para a detecção de infecção no local da queimadura. Alguns dos sinais são escurecimento da região queimada, evolução da necrose local, tecido subcutâneo com coloração esverdeada, aparecimento de vesículas, áreas cicatrizadas e sinais flogísticos próximos à queimadura.

Segundo van Duin et al. (2016), a probabilidade desenvolver Infecção Associada a Hospitalização (IAH) em pacientes queimados foi diretamente proporcional ao tempo de internação, de modo que quase $50 \%$ dos pacientes que permaneceram hospitalizados 80 dias após a admissão tiveram pelo menos uma IAH. As infecções de pele e tecidos moles foram as primeiras a ocorrer, com um tempo médio de três dias após a admissão, seguido por infecções respiratórias (tempo médio de 30 dias após admissão) e infecções por $C$. difficile (tempo médio após admissão de 35,5 dias). As infecções da corrente sanguínea e do trato urinário ocorreram mais tardiamente, com mediana de 41 dias após a admissão. Streptococcus spp., S. aureus, Enterococcus 
spp. e outros patógenos gram-positivos foram geralmente os primeiros a serem encontrados, com tempos médios de isolamento de 02 dias, 03 dias, 09 dias e 6,5 dias, respectivamente. Em contraste, Enterobacteriaceae, Pseudomonas sp. e Acinetobacter $s p$. tenderam a ocorrer mais tardiamente durante a hospitalização, com uma mediana de 11,5 dias, 18 dias e 26 dias, respectivamente. Ainda nesse estudo, hospitalizações de longo prazo levaram ao surgimento de infecções por bactérias multirresistentes. De forma semelhante, Farina Jr, Almeida, Barros, e Martinez (2014) demonstraram que as bactérias grampositivas prevalecem nas duas primeiras semanas de internação hospitalar, enquanto que na terceira e quarta semana há prevalência das gram-negativas. Já após a quarta semana, ocorre o retorno da prevalência dos germes gram-positivos, junto com proliferação de Staphylococcus Aureus resistentes.

Desse modo, segundo Gonella et al. (2014) os exames sequenciais acerca da microbiota dos pacientes queimados no decorrer da internação são importantes para o conhecimento do perfil de colonização momentâneo e para inibir mais rapidamente as infecções nesses pacientes. Segundo Mota et al. (2014) é comum que pacientes queimados recebam antibióticos empíricos, às vezes até sem infecção documentada, de modo que mais de $25 \%$ desses pacientes recebem antibióticos no momento da sua admissão. Sendo assim, o conhecimento precoce de infecção em pacientes com queimadura auxilia no diagnóstico e conduz adequadamente a terapia antimicrobiana.

Wardhana, Djan, e Halim (2017) mostraram que em 52,3\% das colonizações em seu estudo eram causadas por bactérias gram-negativas e apenas $13,7 \%$ por gram-positivas, esses achados foram associados ao fato de que os pacientes selecionados tinham em média 16,47 dias de internamento. O mesmo trabalho ainda mostrou que em uma avaliação mês a mês, por três meses, o padrão de colonização bacteriana na unidade mudou significativamente. Inicialmente, seis principais bactérias (Pseudomonas aeruginosa, Acinetobacter baumannii, Klebsiella pneumoniae, Staphylococcus epidermidis, Enterococcus faecalis, e Enterobacter aerogenes) predominaram em taxas semelhantes, cerca de $14 \%$. No segundo mês, Acinetobacter baumannii (29\%) foi o mais isolado nas amostras, seguido por Klebsiella pneumoniae (25\%) e Pseudomonas aeruginosa (25\%). No terceiro mês, a ocorrência de Klebsiella pneumoniae subiu para 35\% tornando-se a principal bactéria isolada, seguida por Pseudomonas aeruginosa (15\%).

Semelhantemente, Araújo, e Tacla (2020) demonstraram variação do padrão microbiológico em uma mesma instituição de 2011 a 2018. No ano de 2013 houve grande predominância de Pseudomonas sp. No ano de 2014 houve uma queda drástica do número de culturas positivas, e de 2015 a 2018 existiu um aumento progressivo das positividades de culturas, com predomínio de Staphylococcus sp.

Sodré et al. (2015), em seu estudo no Hospital Federal do Andaraí, utilizando das hemoculturas, biópsias de pele, urina, secreção traqueal e lavagem brônquio-alveolar, demonstrou que, nesse período, houve variação da predominância de mircroorganismos causadores de infecção dentro da mesma instituição, evidenciando a necessidade do conhecimento do padrão patogênico para guiar os esquemas antimicrobianos. Além disso, segundo o mesmo autor, é necessário a realização de estudos para esclarecer fatores como resistência bacteriana aos antibióticos, discordâncias nos protocolos de prevenção de infecção em diferentes unidades de queimados, e diferenças na distribuição epidemiológica das infecções no queimado.

\section{Considerações Finais}

A revisão dos 18 artigos selecionados mostrou que, em pacientes queimados, as bactérias mais frequentes em culturas são as gram-negativas em relação às gram-positivas. Em culturas de feridas e de sítios diversos desses pacientes, os gêneros de bactérias mais detectadas foram Pseudomonas, Staphylococcus, Acinetobacter, Proteus, Enterobacter, Klebsiella, Escherichia, Enterococo e Serratia.

Os agentes causadores de infecção identificados neste estudo de revisão sofreram grandes variações de frequência. Trabalhos de revisão de literatura como este são importantes para o entendimento global do comportamento desses patógenos. 
Futuramente, novos estudos podem ajudar a elucidar os motivos dessa variação. O conhecimento destes germes é fundamental para o correto manejo e redução da taxa de mortalidade dos pacientes queimados. Dessa forma, estudos que determinem esses agentes devem ser periodicamente realizados nas instituições hospitalares, para que não haja defasagem das informações ao longo do tempo.

\section{Referências}

American College of Surgions Committee on Trauma. (2018). Advanced Trauma Life Suport - ATLS. 10 ed.

Araújo, A. F., \& Tacla, E. M. (2020). Microbiological profile and antimicrobial resistance profile of patients admitted to the Burn Unit of Hospital Geral "José Pangella" in Vila Penteado in Vila Penteado, Brazil. Revista Brasileira de Cirurgia Plástica, 35(2), 175-181. http://www.dx.doi.org/10.5935/21771235.2020RBCP0030.

Asati, S., \& Chaudhary, U. (2017). Prevalence of biofilm producing aerobic bacterial isolates in burn wound infections at a tertiary care hospital in northern India. Annals of burns and fire disasters, 30(1), 39-42. https://www.ncbi.nlm.nih.gov/pmc/articles/PMC5446907/

Balmelli, B., Sandoval, J., \& Canata, G. (2018). Infecciones en niños quemados internados en el Centro Nacional de Quemados y Cirugías Reconstructivas (CENQUER) Paraguay de Enero 2017 a Enero 2018. Revista de salud publica del Paraguay, 8(2), 45-51. https://dx.doi.org/10.18004/rspp.2018.diciembre.4551.

Cavalcante, I. dos S., Lopes, M. S., Mendes, J. P. S., Techi, L. de C., Lima, D. A., Oliveira, J. E. N., Barbosa, D. L. L., Barros, Y. S. O., Fontenelle, L. F. do V., Santos, L. M. de S. A. dos., \& Teixeira, P. M. G. (2021). Atendimento e manejo de pacientes queimados: Revisão integrativa. Research, Society and Development, 10(7), e0210716308. https://doi.org/10.33448/rsd-v10i7.16308.

Corcione, S., Lupia, T., \& Rosa, F. G. De. (2020). Microbiome in the setting of burn patients: implications for infections and clinical outcomes. Burns \& Trauma, 8, tkaa033. https://doi.org/10.1093/burnst/tkaa033.

Emami, A., Pirbonyeh, N., Keshavarzi, A., Javanmardi, F., Moradi Ghermezi, S., \& Ghadimi, T. (2020) Three Year Study of Infection Profile and Antimicrobial Resistance Pattern from Burn Patients in Southwest Iran. Infect Drug Resist, 13, 1499-1506. https://doi.org/10.2147/IDR.S249160.

El Hamzaoui, N., Barguigua, A., Larouz, S., \& Maouloua, M. (2020). Epidemiology of burn wound bacterial infections at a Meknes hospital, Morocco. New Microbes and New Infections, 38, 100764, https://doi.org/10.1016/j.nmni.2020.100764.

Farina Jr, J. A., Almeida, C. E. F., Barros, M. E. P. M., \& Martinez, R. (2014) Redução da mortalidade em pacientes queimados. Revista Brasileira de Queimaduras, $\quad$ 13(1), 2-5. http://www.rbqueimaduras.com.br/details/188/pt-BR/reducao-da-mortalidade-em-pacientesqueimados\#: :text=A1\%C3\%A9m\%20da\%20utiliza\%C3\%A7ao\%20racional\%20de,com\%20redu\%C3\%A7ao\%20da\%20sua\%20mortalidade.

Forson, O. A., Ayanka, E., Olu-Taiwo, M., Pappoe-Ashong, P. J., \& Ayeh-Kumi, P. J. (2017). Bacterial infections in burn wound patients at a tertiary teaching hospital in Accra, Ghana. Annals of burns and fire disasters, 30(2), 116-120. https://pubmed.ncbi.nlm.nih.gov/29021723/.

Gonella, H. A., Quevedo, F., \& Garbossa, L. C. D. (2014). Colonização bacteriana nas primeiras 24 horas das queimaduras. Revista Brasileira de Queimaduras, 13(2), 99-102. http://rbqueimaduras.org.br/details/203/pt-BR/colonizacao-bacteriana-nas-primeiras-24-horas-das-queimaduras.

Güldoğan, C. E., Kendirci, M., Tikici, D., Gündoğdu, E., \& Yast1, A. Ç. (2017). Clinical infection in burn patients and its consequences. Ulusal travma ve acil cerrahi dergisi = Turkish journal of trauma \& emergency surgery: TJTES, 23(6), 466-471. https://doi.org/10.5505/tjtes.2017.16064.

Gupta, M., Naik, A. K., \& Singh, S. K. (2019). Bacteriological profile and antimicrobial resistance patterns of burn wound infections in a tertiary care hospital. Heliyon, 5(12), e02956. https://doi.org/10.1016/j.heliyon.2019.e02956.

Gragnani, A., Dell'Aquila, A. M., Doi, A. M., Müller, B. R., Lacerda, L. A., Machado, A. M. O., \& Ferreira, L. M. (2014). Perfil microbiológico da unidade de queimaduras da EPM/UNIFESP, São Paulo, Brasil. Revista Brasileira de Cirurgia. Plástica, 29(1), 114-119. http://www.dx.doi.org/10.5935/21771235.2014RBCP0019

Horta, R., Tomaz, D., Egipto, P., \& Silva, A. (2020). The outcome of fungal infections in a burn intensive care unit: a study of 172 patients. Annals of burns and fire disasters, 33(2), 101-106. Retrieved September, 18, from https://pubmed.ncbi.nlm.nih.gov/32913429/.

Latifi, N. A., \& Karimi, H. (2017). Correlation of occurrence of infection in burn patients. Annals of burns and fire disasters, 30(3), 172-176. https://pubmed.ncbi.nlm.nih.gov/29849518/.

Lima, J. S. F., Oliveira, E. A., Araújo, A. C. R. A., \& Oliveira, M. M. (2014). Infecção em crianças com queimadura: Revisão da literatura. Revista Brasileira de Queimaduras, 13(2), 67-75. Recuperado em 18 setembro, 2021, de http://www.rbqueimaduras.com.br/details/198/pt-BR/infeccao-em-criancas-comqueimadura--revisao-da-literatura.

Lima, J. S. F., Oliveira, E. A., Araújo, A. C. R. A., \& Oliveira, M. M. (2016). Risk factors for infection in burn in children and adolescents: a cohort study. Revista Brasileira de Cirurgia Plástica, 31(4), 545-553. http://www.dx.doi.org/10.5935/2177-1235.2016RBCP0079.

Mater, M. E., Yamani, A. E., Aljuffri, A. A., \& Binladen, S. A. (2020). Epidemiology of burn-related infections in the largest burn unit in Saudi Arabia. Saudi medical journal, 41(7), 726-732. https://doi.org/10.15537/smj.2020.7.25141.

Mélega, J. M., Viterbo, F., \& Mendes, F. H. (2011). Cirurgia plástica: os princípios e a atualidade. Guanabara Koogan. 
Mota, W. M., Araújo, C. A. L., Oliveira, A. M. R. R., Gomez, D. S., Silva Junior, J. M., \& Gemperli, R. (2014). Critérios diagnósticos de infecção no paciente queimado. Revista Brasileira de Queimaduras, 13(3), 130-135. http://rbqueimaduras.org.br/details/211/pt-BR/criterios-diagnosticos-de-infeccao-no-pacientequeimado.

Ramakrishnan, M., Putli Bai, S., \& Babu, M. (2016). Study on biofilm formation in burn wound infection in a pediatric hospital in Chennai, India. Annals of burns and fire disasters, 29(4), 276-280. https://pubmed.ncbi.nlm.nih.gov/28289362/.

Rempel, L. C. T., Tizzot, M. R. P. A., \& Vasco, J. F. M. (2011). Incidência de infecções bacterianas em pacientes queimados sob tratamento em hospital universitário de Curitiba. Revista Brasileira de Queimaduras,10(1), 3-9. http://www.rbqueimaduras.com.br/details/56/pt-BR/incidencia-de-infeccoesbacterianas-em-pacientes-queimados-sob-tratamento-em-hospital-universitario-de-curitiba

Roham, M., Momeni, M., Saberi, M., Kheirkhah, R., Jafarian, A., \& Rahbar, H. (2017). Epidemiologic analysis of central vein catheter infection in burn patients. Iranian journal of microbiology, 9(5), 271-276. https://www.ncbi.nlm.nih.gov/pmc/articles/PMC5748445/.

Rosanova, M. T., Voto, C., Highton, E., Carnovale, S., Caracciolo, B., Tramonti, N., Lema, J., Álvarez, V., Villasboas, R. M., Laborde, S., \& Basilico, H. (2019). Osteomielitis fúngica en niños quemados. Med. Infant, 26(3), 272-275. https://pesquisa.bvsalud.org/portal/resource/pt/biblio-1026763.

Sala, L. P., Lima, N. L., Simioni, P. U., \& Ugrinovich, L. A. (2016) Principais patógenos envolvidos em casos de sepse em pacientes queimados: uma revisão de literatura. Revista Brasileira de Queimaduras, 15(3), 164-168. http://www.rbqueimaduras.com.br/details/313/pt-BR/principais-patogenos-envolvidos-emcasos-de-sepse-em-pacientes-queimados--uma-revisao-de-literatura.

Silva, A. V., Tavares, D. S., Tavares, P. A. M., \& Santos, C. O. (2020). Terapias aplicadas no tratamento das lesões por queimaduras de terceiro grau e extensão variável: revisão integrativa. Medicina (Ribeirão Preto), 53(4), 456-463. https://doi.org/10.11606/issn.2176-7262.v53i4p456-463.

Sodré, C. N. S., Serra, M. C. V. F., Rios, J. A. S., Cortorreal, C. G., Maciera, L., \& Morais, E. N. (2015). Perfil de infecção em pacientes vítimas de queimadura no Hospital Federal do Andaraí. Revista Brasileira de Queimaduras, 14(2), 109-112. http://www.rbqueimaduras.com.br/details/249/pt-BR/perfilde-infeccao-em-pacientes-vitimas-de-queimadura-no-hospital-federal-do-andarai.

Souza, L. R. P. de., Lima, M. F. A. B., Dias, R. O., Cardoso, E. G., Briere, A. L., \& Silva, J. O. (2021). O tratamento de queimaduras: uma revisão bibliográfica. Brazilian Journal of Development, 7(4), 37061-37074. https://doi.org/10.34117/bjdv7n4-257.

Vaez, H., \& Beigi, F. (2016). Antibiotic susceptibility patterns of aerobic bacterial strains isolated from patients with burn wound infections. Germs, 6(1), 3436. https://doi.org/10.11599/germs.2016.1087.

van Duin, D., Strassle, P. D., DiBiase, L. M., Lachiewicz, A. M., Rutala, W. A., Eitas, T., Maile, R., Kanamori, H., Weber, D. J., Cairns, B. A., Napravnik, S., \& Jones, S. W. (2016). Timeline of health care-associated infections and pathogens after burn injuries. American journal of infection control, 44(12), 15111516. https://doi.org/10.1016/j.ajic.2016.07.027.

Wardhana, A., Djan, R., \& Halim, Z. (2017). Bacterial and antimicrobial susceptibility profile and the prevalence of sepsis among burn patients at the burn unit of Cipto Mangunkusumo Hospital. Annals of burns and fire disasters, 30(2), 107-115. https://pubmed.ncbi.nlm.nih.gov/29021722/. 